\title{
OPTICAL PROPERTIES - AND MICROSTRUCTURE OF InGaN GROWN BY MOLECULAR BEAM EPITAXY
}

\author{
T. Böttcher, S. Einfeldt, S. Figge, V. Kirchner, D. Hommel
}

Institute of Solid State Physics, University of Bremen

P.O. Box 330 440, 28334 Bremen, Germany

H. SELKE, P.L. RYDER

Institute of Materials Physics and Structural Research, University of Bremen P.O. Box 330 440, 28334 Bremen, Germany

F. Bertram, T. Riemann, J. Christen

Institute of Experimental Physics, University of Magdeburg

P.O. Box 4120, 39016 Magdeburg, Germany

\section{U. LUNZ AND C.R. BECKER}

Institute of Experimental Physics III, University of Würzburg

Am Hubland 3, 97074 Würzburg, Germany

The luminescence of $\operatorname{In}_{x} \mathrm{Ga}_{1-x} \mathrm{~N}$ is studied for thick epitaxial layers and quantum wells. Using spatially resolved cathodoluminescence spectroscopy the commonly observed broad integral photoluminescence spectra. were found to result from spectral and lateral inhomogeneous emission across the samples. Moreover, the integral photoluminescence and absorption spectra show different temperature dependences. The effects can be explained assuming fluctuations of the composition associated with a variation of the band gap.

PACS numbers: $68.35 . \mathrm{Dv}, 68.65 . \mathrm{g}, 78.20 .-\mathrm{e}, 78.55 .-\mathrm{m}, 78.55 . \mathrm{Cr}$

\section{Introduction}

The development of blue light emitting diodes [1] and of laser diodes [2] has raised a lot of interest in the properties of GaN-based III-V nitrides. Particularly $\mathrm{In}_{x} \mathrm{Ga}_{1-x} \mathrm{~N}$ is important for the use in the active region of these devices as the band gap of this material can be varied over nearly the whole spectral range [3]. However, the growth of $\operatorname{In}_{x} \mathrm{Ga}_{1-x} \mathrm{~N}$ is hampered by different thermal stabilities and the large lattice mismatch of the two binaries InN and GaN. The latter leads to 
a miscibility gap [4], which can cause fluctuations of the In content across the film. Evidence for these fluctuations has been reported recently for thick $\operatorname{In}_{x} G a_{1-x} \mathrm{~N}$ films [5] as well as for quantum well structures [6]. As the decomposition of InN starts at approximately $630^{\circ} \mathrm{C}$ whereas $\mathrm{GaN}$ is stable up to $850^{\circ} \mathrm{C}[7]$ loss of In from the film during growth may occur due to the thermal dissociation of In-N bonds. This leads to the formation of In droplets on the surface which is an additional source for the observed fluctuations of the In content $[8,9]$.

In recent papers we reported on growth issues and on the microstructure of thick $\ln _{x} \mathrm{Ga}_{1-x} \mathrm{~N}$ films in view of the variation of the local and the average composition $[9,10]$. In this study we focus on the influence of fluctuations found in thick $\operatorname{In}_{x} \mathrm{Ga}_{1-x} \mathrm{~N}$ films and quantum wells on the optical properties of these structures.

\section{Experimental}

All samples have been grown on $c$-plane sapphire substrates in a standard MBE system using conventional effusion cells for Ga and In. Nitrogen was supplied through a radio frequency (rf) plasma source allowing growth rates of approximately $600 \mathrm{~nm} / \mathrm{h}$. The samples were characterized utilizing high-resolution $\mathrm{X}$-ray diffraction (HRXRD), cathodoluminescence spectroscopy (CL), absorption spectroscopy, and photoluminescence spectroscopy (PL) at different temperatures and excitation densities. The CL measurements were performed in a modified scanning electron microscope where the samples are excited by a focused electron beam. This electron beam is scanned over the area of interest and luminescence spectra are recorded at each point [11].

\section{Results and discussion}

CL images reveal an inhomogeneous distribution of the luminescence emission across the sample. An example of an intensity image which displays the peak intensity at each sampling point is shown in Fig. 1 for a $1000 \mathrm{~nm}$ thick $\operatorname{In}_{x} \mathrm{Ga}_{1-x} \mathrm{~N}$ film grown on a $30 \mathrm{~nm} \mathrm{GaN}$ buffer. The bright and dark regions of the image correspond to high and low intensity, respectively. Typical structure sizes of the local emission found in the image are in the range of 100 to $500 \mathrm{~nm}$. The intensity can be correlated to the local emission wavelength as illustrated by the two local spectra displayed in Fig. 1 which were recorded from the areas indicated in the CL image. It should be noted that the local spectra feature at least one rather narrow peak with a full width at half maximum (FWHM) in the range from $55 \mathrm{meV}$ to $130 \mathrm{meV}$. This value is significantly lower than the commonly observed broad integral PL emission with a FWHM of approximately $350 \mathrm{meV}$. The regions with the longer peak emission wavelength usually exhibit the higher peak intensity. This has been further confirmed by the comparison of intensity images with images of the peak emission wavelength. The behaviour can be explained assuming a lateral variation of the band gap as sketched in Fig. 2. It is probably caused by local fluctuations of the In content which were found in several samples utilizing energy dispersive X-ray (EDX) analysis [9]. The band gap variation causes the relaxation of excited charge carriers into local potential minima leading to the observed localized emission. As the luminescence is very intense in $\mathrm{In}_{x} \mathrm{Ga}_{1-x} \mathrm{~N}$ in spite of a 


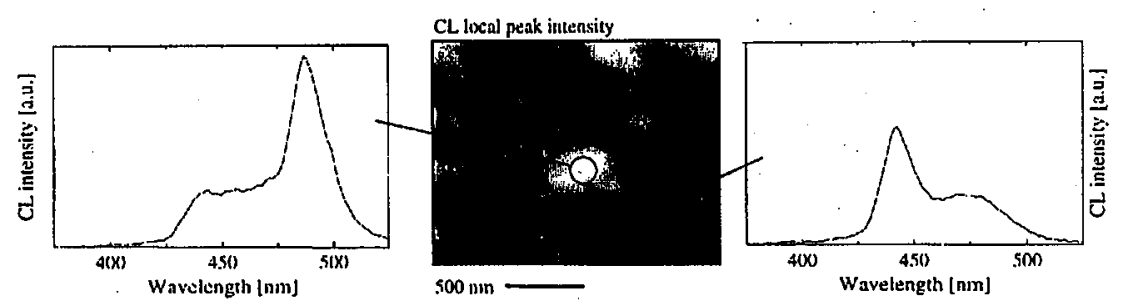

Fig. 1. Cathodoluminescence spectroscopy of a thick $\operatorname{In}_{x} \mathrm{Ga}_{1-x} \mathrm{~N}$ film at $5 \mathrm{~K}$. A mapping of the peak intensity and two local spectra from the indicated locations on the sample are shown.

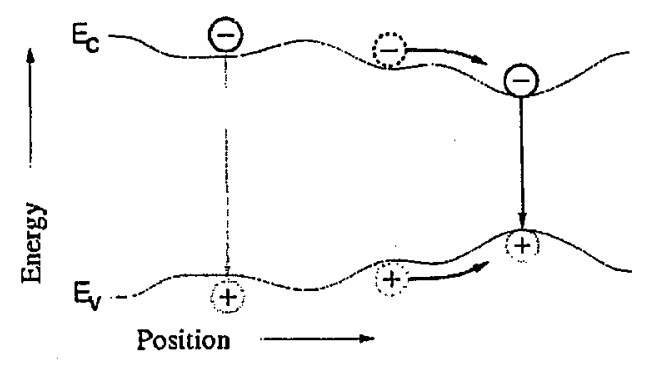

Fig. 2. Proposed model for the recombination mechanism in $\operatorname{In}_{x} \mathrm{Ga}_{1-x} \mathrm{~N}$ due to lateral inhomogeneities of the band edge.

high dislocation density in the samples this localization mechanism appears to be fairly efficient.

$\mathrm{In}_{x} \mathrm{Ga}_{1-x} \mathrm{~N}$ is usually characterized by a strong Stokes shift in the order of $300 \mathrm{meV}$ which can be explained by the proposed model of the band edge variation. The measured absorption edge represents a simple average of the local absorption coefficient across the sample volume, i.e., it yields an a verage band gap. Contrarily, the luminescence occurs in local potential minima and thus probes the minimum band gap in a certain area. Figure 3a contains the position of the absorption edge and the PL emission maximum in dependence on the temperature for a $100 \mathrm{~nm}$ thick $\operatorname{In}_{x} \mathrm{Ga}_{1-x} \mathrm{~N}$ film grown on a $30 \mathrm{~nm}$ GaN buffer. The temperature dependence of the absorption edge can be described with the equation proposed by Vina et al. [12] using similar parameters as published for GaN [13]. In spite of this the position of the PL peak is not coupled to the band gap and exhibits a significantly stronger shift towards lower energies which represents an increase in the Stokes shift. The reason for this is the rising kinetic energy of the charge carriers with temperature enabling them to overcome potential barriers more effectively and relax into local potential minima. This fits well to the short-scale inhomogeneities of the In content up to a few percent as this would cause band edge variations of approximately $\pm 20 \mathrm{meV}$ and the thermal energy ranges up to $25 \mathrm{meV}$. 

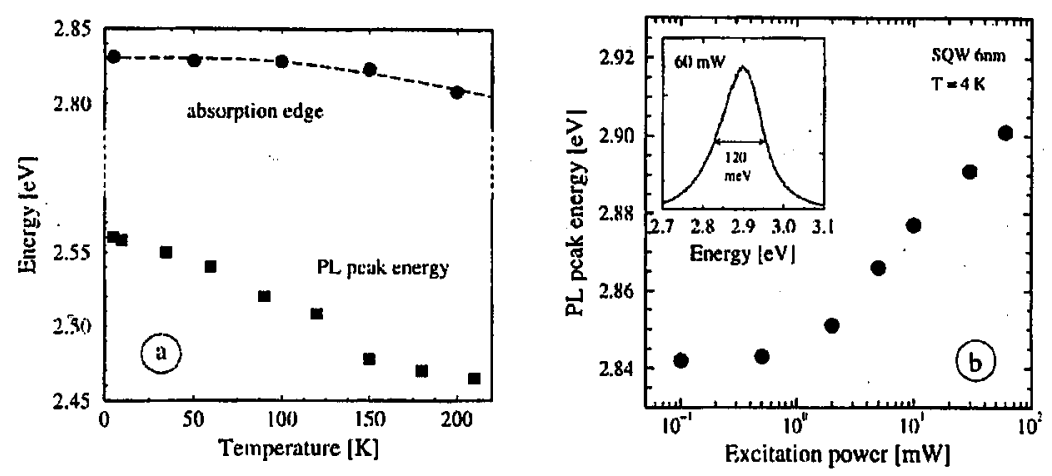

Fig. 3. Optical properties of $\mathrm{In}_{x} \mathrm{Ga}_{1-x} \mathrm{~N}$ films and quantum structures. (a) Temperature dependence of the absorption edge and the PL maximum of a $100 \mathrm{~nm}$ thick film. (b) The position of the PL peak in dependence on the excitation power for a $6 \mathrm{~nm}$ thick $\mathrm{In}_{x} \mathrm{Ga}_{1-x} \mathrm{~N} / \mathrm{GaN}$ SQW.

Band gap fluctuations are not only observed in thick $\mathrm{In}_{x} \mathrm{Ga}_{1-x} \mathrm{~N}$ layers but also in $\operatorname{In}_{x} \mathrm{Ga}_{1-x} \mathrm{~N} / \mathrm{GaN}$ quantum wells. The joint density of states in quantum well structures is assumed to be Gaussian broadened on the low energy side due to quantum dot-like structures. As a consequence the luminescence should be strongly affected by the excitation density as has been confirmed by the investigation of the electroluminescence of LED's [14]. The dependence of the PL peak energy on the excitation power is displayed in Fig. $3 \mathrm{~b}$ for a $6 \mathrm{~nm}$ thick single quantum well (SQW). At low excitation power all charge carriers can thermalize into potential minima such that the spectral position of the emission is nearly power independent. With higher excitation densities the potential minima are saturated with carriers and higher' states are increasingly filled up corresponding to a Burnstein-Moss shift of the PL peak towards higher energies.

\section{Summary}

In conclusion, a spatially resolved analysis of the luminescence of thick $\mathrm{In}_{x} \mathrm{Ga}_{1-x} \mathrm{~N}$ was performed utilizing high resolution CL spectroscopy. Based on these observations and on EDX measurements performed earlier the composition and the luminescence are shown to be inhomogeneous in this material. A model for the recombination mechanism in a material with varying band gap can qualitatively explain the commonly observed optical properties like the huge and temperature dependent Stokes shift as well as the excitation dependence of the SQW-PL.

\section{References}

[1] S. Nakamura, M. Senoh, N. Iwasa, S. Nagahama, Jpn. J. Appl. Phys. 34, L797 (1995).

[2] S. Nakamura, M. Senoh, S. Nagahama, N. Iwasa, T. Yamada, T. Matsushita, H. Kiyoko, Y. Sugimoto, Jpn. J. Appl. Phys. 35, L74 (1996). 
[3] S. Strite, H. Morkoc, J. Vac. Sci. Technol. B 10, 1237 (1992).

[4] I-hsiu Ho, G.B. Stringfellow, J. Cryst. Growth 178, 1 (1997).

[5] R. Singh, D. Doppalapudi, T.D. Moustakas, L.T. Romano, Appl. Phys. Lett. 70, 1089 (1997).

[6] M.D. McCluskey, L.T. Romano, B.S. Krusor, D.P. Bour, N.M. Johnson, S. Brennan, Appl. Phys. Lett. 72, 1730 (1998).

[7] O. Ambacher, M.S. Brandt, R. Dimitrov, T. Metzger, M. Stutzmann, R.A. Fisher, A: Miehr, A. Bergmaier, G. Dollinger, J. Vac. Sci. Technol. B 14, 3532 (1996).

[8] M.A.L. Johnson, W.C. Hughes, W.H. Rowland, Jr., J.W. Cook, Jr., J.F. Schetzina, M. Leonard, H.S. Kong, J.A. Edmond, J.. Zavada, J. Cryst. Growth 175/6, 72 (1997).

[9] H. Selke, T. Böttcher, S. Einfeldt, F. Bertram, V. Kirchner, J. Christen, D. Hommel, submitted to Appl. Phys. Lett.

[10] T. Böttcher, S. Einfeldt, S. Figge, V. Kirchner, D. Hommel, H. Selke, P.L. Ryder, submitted to Appl. Phys. Lett.

[11] J. Christen, M. Grundnamm, D. Bimberg, J. Vac. Sci. Technol. B 9, 2358 (1991).

[12] L. Vina, S. Logothedis, M. Cardona, Phys. Rev. B 30, 1979 (1983).

[13] J. Petalas, S. Logothetidis, S. Boultadakis, M. Alouani, J.M. Wills, Phys. Rev. Lett. 52, 8082 (1995).

[14] P.G. Eliseev, P. Perlin, J. Lee, M. Osiński, Appl. Phys. Lett. 71, 569 (1997). 